\title{
Yeats et la scène, l'acteur et sa voix à l'Abbey Theatre de Dublin
}

\section{Jacqueline Genet}

\section{(2) OpenEdition \\ 1 Journals}

\section{Édition électronique}

URL : http://journals.openedition.org/etudesirlandaises/4894

DOI : 10.4000/etudesirlandaises.4894

ISSN : 2259-8863

\section{Éditeur}

Presses universitaires de Caen

\section{Édition imprimée}

Date de publication : 15 juin 2016

Pagination : 185-187

ISBN : 978-2-7535-5091-9

ISSN : 0183-973X

\section{Référence électronique}

Jacqueline Genet, « Yeats et la scène, l'acteur et sa voix à l'Abbey Theatre de Dublin », Études irlandaises [En ligne], 41-1 | 2016, mis en ligne le 15 juin 2016, consulté le 24 septembre 2020. URL : http://journals.openedition.org/etudesirlandaises/4894; DOI : https://doi.org/10.4000/ etudesirlandaises.4894

\section{(c) (1) ()}

Études irlandaises est mise à disposition selon les termes de la Licence Creative Commons Attribution - Pas d'Utilisation Commerciale - Partage dans les Mêmes Conditions 4.0 International. 
avec des perspectives plus larges puisque, comme Yeats, les poètes contemporains abordent les contraintes historiques, politiques et religieuses pour les dépasser. Les confluences mises à jour concernent Boland, Longley, Mahon ou Heaney. En se penchant sur les proximités entre les écrits tardifs de Yeats et de T. S. Eliot, Jed Esty s'interroge sur les points communs entre les deux poètes, en portant l'accent sur des cycles de vie, incluant mort et résurrection, voire une écriture du déclin de la civilisation. Le point de rencontre des deux écrivains pourrait être leur propre vieillissement et celui d'une civilisation, où une dimension créatrice permettrait peut-être d'effacer la composante historique du temps. Au vu de la richesse des contributions, il ne fait aucun doute que Yeats and Afterwords figurera en bonne place parmi les ouvrages utiles aux spécialistes de W. B. Yeats.

Thierry Dubost

Université de Caen-Normandie

Pierre Longuenesse, Yeats et la scène, l'acteur et sa voix à l'Abbey Theatre de Dublin, Villeneuve-d'Ascq, Presses universitaires du Septentrion, coll. «Irlande », 2015, 224 pages, ISBN 978-2-7574-0851-3.

Yeats et la scène, l'acteur et sa voix à l'Abbey Theatre de Dublin s'inscrit dans le courant d'intérêt porté au théâtre de W. B. Yeats dont l'initiateur Ronald Schuchard est cité en exergue. Le contexte irlandais et européen y est envisagé. Yeats rejette et le théâtre nationaliste irlandais reflet des "réalités rurales " ou des "luttes militantes ", et les " conventions du théâtre victorien ". Il s'en prend aux productions irlandaises du Queens, refuse "la surcharge des détails décoratifs ", "les costumes qui tuent l'imagination " et l'agitation injustifiée des acteurs des théâtres commerciaux. Le contexte culturel, littéraire et musical est évoqué à travers Arthur Symons. "La dimension magique au cœur de l'événement théâtral " permet de rapprocher Yeats et Antonin Artaud. Mallarmé est cité plusieurs fois ainsi qu'Ezra Pound qui découvre le nô et pour qui la musique est "l'essence même de l'écriture poétique. " Des références à Arnold Schönberg et à Pierre Boulez accompagnent l'analyse du « sprechgesang ».

La complémentarité parole-musique-danse est au cœur du livre. L'accent porte sur les mots - sonorité, rythme - et leur passage sur scène par la voix de l'acteur, sur les mouvements qui doivent être simplifiés, sacralisés, avec une interrelation entre travail corporel et composition vocale, celle des acteurs ou du chœur. La musique de At the Hawk's Well "s'apparente [...] plus à un livret qu'à un texte dramatique ", et justifie l'analogie avec « certaines expériences de mélodrame musical ", terme défini 
au chapitre 4 qu'il aurait été judicieux d'expliquer dès son premier emploi. Voix, gestes, musique, sources de "révélation ", créent une " cérémonie ritualisée ". En sappuyant sur The Only Jealousy of Emer, P. Longuenesse montre que les parties parlées sont le lieu du réel, alors que le chant se situe dans un mode visionnaire. "Diseurs » ou chanteurs, les mouvements de leur corps rendent visible l'invisible; l'auteur s'interroge : ces pièces sont-elles des représentations ou des révélations? Si la parole parlée-chantée devient " une psalmodie proche de la prière », la représentation un "rituel orphique ", les sources sont " autant profanes que sacrées ». N'auraitil pas été préférable de regrouper l'ouverture du livre sur " Yeats et la religion » et les analyses des pièces qui en sont l'illustration?

Le thème récurrent des pièces-nô de Yeats est l'ingérence de l'au-delà, voyage du héros vers la mort, rencontre avec des fantômes, dans " un espace-seuil entre le monde des vivants et celui des esprits ", questionnement sur l'incarnation et la vie après la mort. La voix qui parle avec les morts est celle de l'acteur, seule ou accompagnée de musique et de danse, manifestation d'une "puissance d'outretombe ». Si P. Longuenesse s'interroge à juste titre sur les croyances de Yeats, il refuse " l'institution religieuse " et les " dogmes "; n’aurait-il pas été préférable d'évoquer d'emblée son syncrétisme? Parler de théologie ne semble guère possible sauf, comme indiqué par l'auteur, dans Calvary et The Resurrection. Le credo de Yeats est cette "Unity of Being » définie à maintes reprises.

En quête d'un acteur nouveau pour une scène renouvelée, Yeats préfère aux acteurs professionnels les amateurs qu'il peut former à la diction psalmodiée. Il consacre à Florence Farr et Frank Fay, acteurs et metteurs en scène lors des années de l'Irish Literary Theatre, plusieurs pages et de longues notes. Leur parole est « le vecteur central de la théâtralité ", leur psalmodie rythmée soutenue par le psaltérion d'Arnold Dolmetsch dont les cordes sont accordées en fonction de la voix. Une succession de rondes sans mesure peut former la partition, ainsi le chant "The Four Rivers" composé par Florence Farr. Frank Fay et son frère William collaborent avec Yeats à partir de 1902; ils entendent ne pas sacrifier texte et parole « au profit d'effets théâtraux inutiles et défendre un répertoire national ». Pour mesurer l'enjeu de l'interprétation, P. Longuenesse, après des jugements élogieux, expose les échecs, de moins bonnes prestations incitant le public à quitter la salle. Quand Frank Fay s'endort sur scène à la première de The Unicorn from the Stars, la rupture est consommée. Les deux frères démissionnent en janvier 1908 et, en 1912, Florence Farr part à Ceylan. Yeats est alors en quête d'acteurs d'exception; Mrs. Patrick Campbell ne sera qu'une solution éphémère. Avec le nô, il trouve en Michio Ito, danseur japonais dont il admire le génie du mouvement, une alliance parfaite avec la pensée : "Il ne s'évadait que pour habiter [...] les profondeurs de l'esprit ", écrit-il. Sa rencontre avec Ninette de Valois en 1927 sera également fructueuse; il compose pour elle Fighting the Waves qu'elle interprète en 
1928 avec les masques d'Hildo Van Krop et la musique de George Antheil; faut-il pour autant comparer les mégaphones utilisés pour la pièce à ceux des séances à la Golden Dawn? Il fut difficile à Yeats d'établir l'empathie nécessaire entre scène et public. At the Hawk's Well et Fighting the Waves n'eurent pas le succès escompté en partie parce que le public, habitué au théâtre de l'époque, n'était pas initié au nô.

Ce livre témoigne d'une excellente connaissance du théâtre de Yeats; on peut regretter que le rôle déterminant de Gordon Craig ne soit que rapidement mentionné, même si son importance est évoquée dans d'autres écrits. P. Longuenesse connaît parfaitement les problèmes posés par la représentation. Ses analyses minutieuses seront appréciées des metteurs en scène. Réjouissons-nous aussi de l'annonce de futures publications.

Jacqueline Genet

Université de Caen Normandie

Anthony Roche, The Irish Dramatic Revival 1899-1939, London, Bloomsbury, 2015, 272 p., ISBN : 978-1-4081-7528-6.

Anthony Roche's new book provides a compact history of the Irish dramatic movement from the first year of the Irish Literary Theatre, initiated by Yeats, Lady Gregory and Edward Martyn, to Yeats's death. Thus Yeats is construed as the tutelary figure of what Roche refers to as the "Irish dramatic revival", yet the book usefully opens up the canon and incorporates discussions of playwrights not often mentioned in the context of the Revival, such as G.B. Shaw and Teresa Deevy. It also broadens the scope to look at those theatrical experiments which took place on other stages than that of the Irish National Theatre, giving ample space to Yeats's experiments with dramaturgical arrangements which were intended for intimate spaces in the 1910s and 20s, and to such initiatives as the Dublin Drama League and the beginnings of the Gate Theatre.

The first chapter, "the Late Nineteenth Century", sets the context for the emergence of the dramatic movement. Commenting on the influence of Douglas Hyde's pioneering work as translator and scholar of the native Irish literary tradition, Roche makes the decisive point that "there was no native Irish drama to develop", so that a canon had to be created to refute the stage Irish stereotypes conveyed on the English stage and in the melodramas of Boucicault, which had been immensely popular but were violently rejected by the intellectual elite who took up the task of representing the "authentic" Ireland during the Revival. The chapter situates Wilde and Shaw as the contemporary luminaries of the Anglo- 